$$
\begin{aligned}
& \text { BNL }-66930 \\
& \text { CAP-262-Muon-99R } \\
& \text { Informal Report }
\end{aligned}
$$

\title{
A Study of the Edge Field Effect Using Mafia Simulations Conformal Mapping
}

\author{
Yongxiang Zhao and Haipeng Wang \\ Brookhaven National Laboratory
}

October 1999 


\title{
A STUDY OF THE EDGE FIELD EFFECT USING MAFIA SIMULATIONS AND CONFORMAL MAPPING*
}

\author{
Yongxiang Zhao, Haipeng Wang \\ Physics Department, Bldg. 901-A \\ Brookhaven National Laboratory, Upton, NY 11973
}

\begin{abstract}
It was found that the measured $Q$ value of a tape resonator has a large difference with that calculated with MAFIA. This difference is recognized to be mainly due to the error in the simulation, which is unable to handle a sharp edge. This note addresses the 2-dimensional MAFIA simulation and conformal mapping. It shows the error could be as high as $60 \%$. Reasonable caution should be exercised in the use of MAFLA, and other codes as well, in calculating $Q$ value if a sharp edge exists in the problem.
\end{abstract}

\section{INTRODUCTION}

We use two parallel beryllium tapes to form a TEM-mode resonator to test its $Q$ and its conductivity. The experiment shows a discrepancy of the measured $Q$ values and that calculated by MAFIA ${ }^{[1,2]}$ For example, the $Q$ of copper tape resonator was tested to be around 1950 at room temperature, while MAFIA gives 3520. Though the former includes the loss of dielectric etc., while the later not, there is still too much difference between each other.

Indeed, there are many factors that influence the equipment and its accuracy. The measured data with a network analyzer are not precise enough due to noise, external loss of coupler, the interference of unexpected mode, intrinsic error of the instrument, system error, etc. The geometry of the real setup is not exactly coincident with those in the simulation. The imperfection of the material as well as its surface contamination may cause higher loss than expected, so on and so forth. However, none of them is likely to cause such a large discrepancy.

Is the result of MAFIA precise enough? The loss accounted by MAFIA is based on the magnetic field magnitude at each mesh point, which is the average to the extent of the grid, while the real loss is proportional the field square. Besides, the values of magnetic field on the metal surface are zero on the MAFIA solution; MAFIA adopts the values on the nearest mesh point for calculating loss. Usually it is less than the value on the surface. Thus the resultant loss is always underestimate.

We tried to increase the number of mesh points to improve the accuracy. However, the 3dimensional simulation needs too many mesh points and too much operation time as well. For moderate mesh density the simulation takes several hours of cpu time. A reduction of mesh size to a half means an increment of mesh points to 8 times, and the cpu time may be 20 times or more. So it is not practical.

\footnotetext{
- This work was supported by the US DOE under Contract No. DE-AC02-98CH10886
} 
The loss of a tape resonator is dominantly concentrated in the center part of the tape, where the current is about maximum. In this part, the field pattern should be the same as in a 2dimensional case; i.e. the case that the tape is extended infinitive. The field pattern near the edge will deviate from the 2-dimensional case, but there is little current thus little loss occurs. 2dimensional simulation needs far less mesh points and is possible to get much precise result. To this end, at least we can estimate how much the error due to a coarse mesh.

It is expected that the more dense the mesh, the more precise the result. Unfortunately, this is not true. On the contrary, the higher the density, the higher the noise, and then the accuracy indicated becomes too poor to be reliable.

Eventually, we realized that the error is due to the sharp edge, which is a singularity that a code can hardly handle. An analytical analysis using conformal mapping was carried out.

\section{TWO-DIMENSIONAL SIMULATION}

As mentioned above, the 3-dimensional simulation via MAFIA resulted in an overestimated $Q$ value (the loss is underestimated). In order to evaluate the overestimation, 2-dimensional simulation was carried out. First, an example (tp423) had with the same mesh structure (nonuniform) as that of 3-d code on the cross section at the center of the tape. It is conceivable that it will cause roughly the same overestimation on $Q$. Thus it is regarded as a reference for further dense mesh cases, i.e. its $Q$ ratio is $100 \%$. It is shown on the first row of Table 1.

Table. 1

\begin{tabular}{|c|c|c|c|c|c|c|c|c|}
\hline & Program & $\begin{array}{c}\text { Tape } \\
\text { Thickness }\end{array}$ & $\begin{array}{c}\text { Total Mesh } \\
\text { Points }\end{array}$ & $\begin{array}{c}\text { Auto- } \\
\text { mesh }^{1}\end{array}$ & $\begin{array}{c}\text { \# of inner } \\
\text { Lines }^{2}\end{array}$ & $\begin{array}{c}\text { Q } \\
\text { Ratio }\end{array}$ & Accuracy $^{3}$ \\
\hline 1 & tp423 & $0(\mathrm{~mm})$ & 825 & no & 4 & 3380 & $100 \%$ & $0.58 \%$ \\
\hline 2 & tp423b & 0 & 3072 & no & 8 & 3162 & $93.5 \%$ & $1.7 \%$ \\
\hline 3 & tp423c & 0 & 11844 & no & 16 & 2895 & $85.6 \%$ & $7.8 \%$ \\
\hline 4 & tp423d & 0 & 38844 & no & 32 & & & $* 80 \%$ \\
\hline 5 & tp426a & 0.15 & 7020 & no & 11 & 2793 & $82.6 \%$ & $6.0 \%$ \\
\hline 6 & tp428a & 0.15 & 5112 & yes & 4 & 3356 & $99.3 \%$ & $2.2 \%$ \\
\hline 7 & tp428b & 0.15 & 20022 & yes & 8 & 3087 & $91.3 \%$ & $1.4 \%$ \\
\hline 8 & tp428c & 0.15 & 80089 & yes & 16 & 2875 & $85.1 \%$ & $4.5 \%$ \\
\hline 9 & tp428d & 0.15 & 107406 & yes & 32 & 2725 & $80.6 \%$ & $* 15 \%$ \\
\hline 10 & tp428e & 0.15 & 128304 & yes & 40 & 2070 & $61.1 \%$ & $* 26 \%$ \\
\hline
\end{tabular}

2. Number of mesh lines inside the tape gap.

3. Accuracy given by MAFIA is a measure of the consistency of the computation with Maxwell equation, not the error of the result. The marker * indicates that the result is not precise.

As shown on the sequent rows, the denser the mesh, the lower the $Q$. However, Further densification results in too much error ( $4^{\text {th }}$ row). Then a real thickness of $0.15 \mathrm{~mm}$ was chosen. Row 5 shows its result. But a denser mesh also cause larger error. We then chose "automesh", that the mesh established by MAFIA itself, to see if it could get more precise result. Unfortunately, the similar results were obtained as shown on row 6 to 10 . 


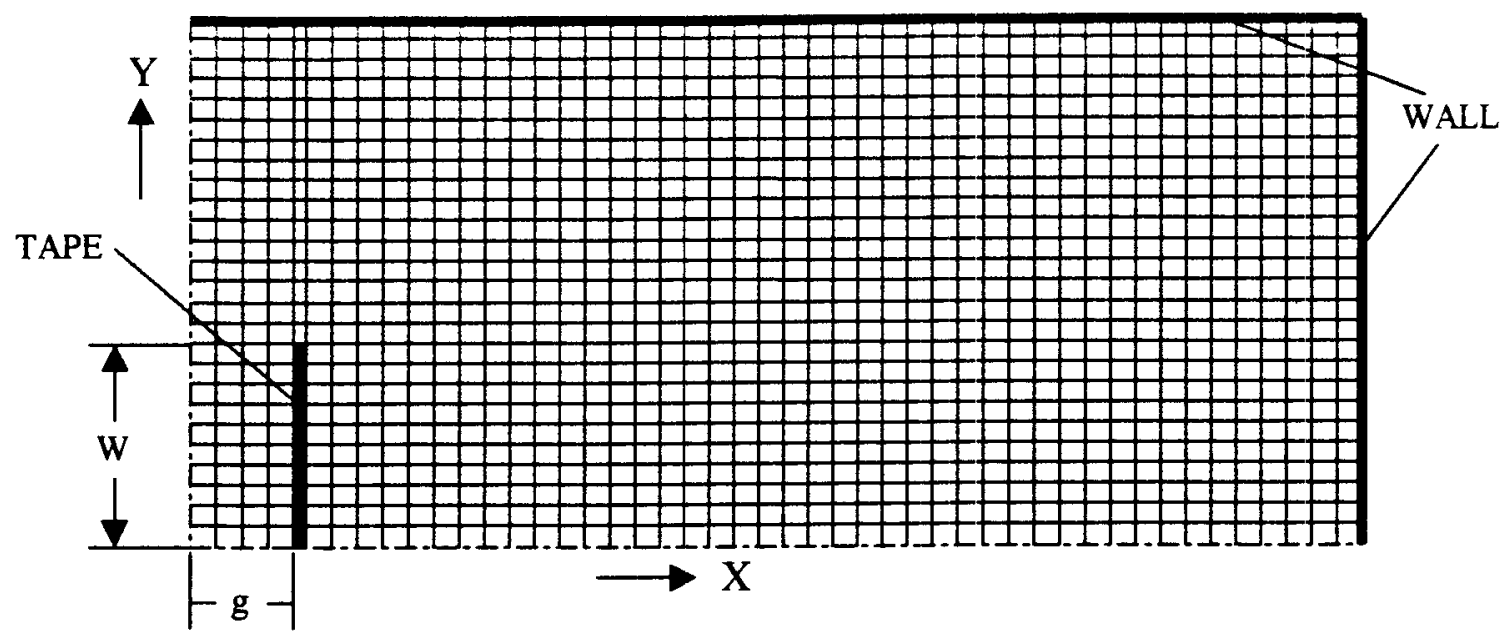

Fig. 1 The mesh structure with 4 lines inside the tape gap.
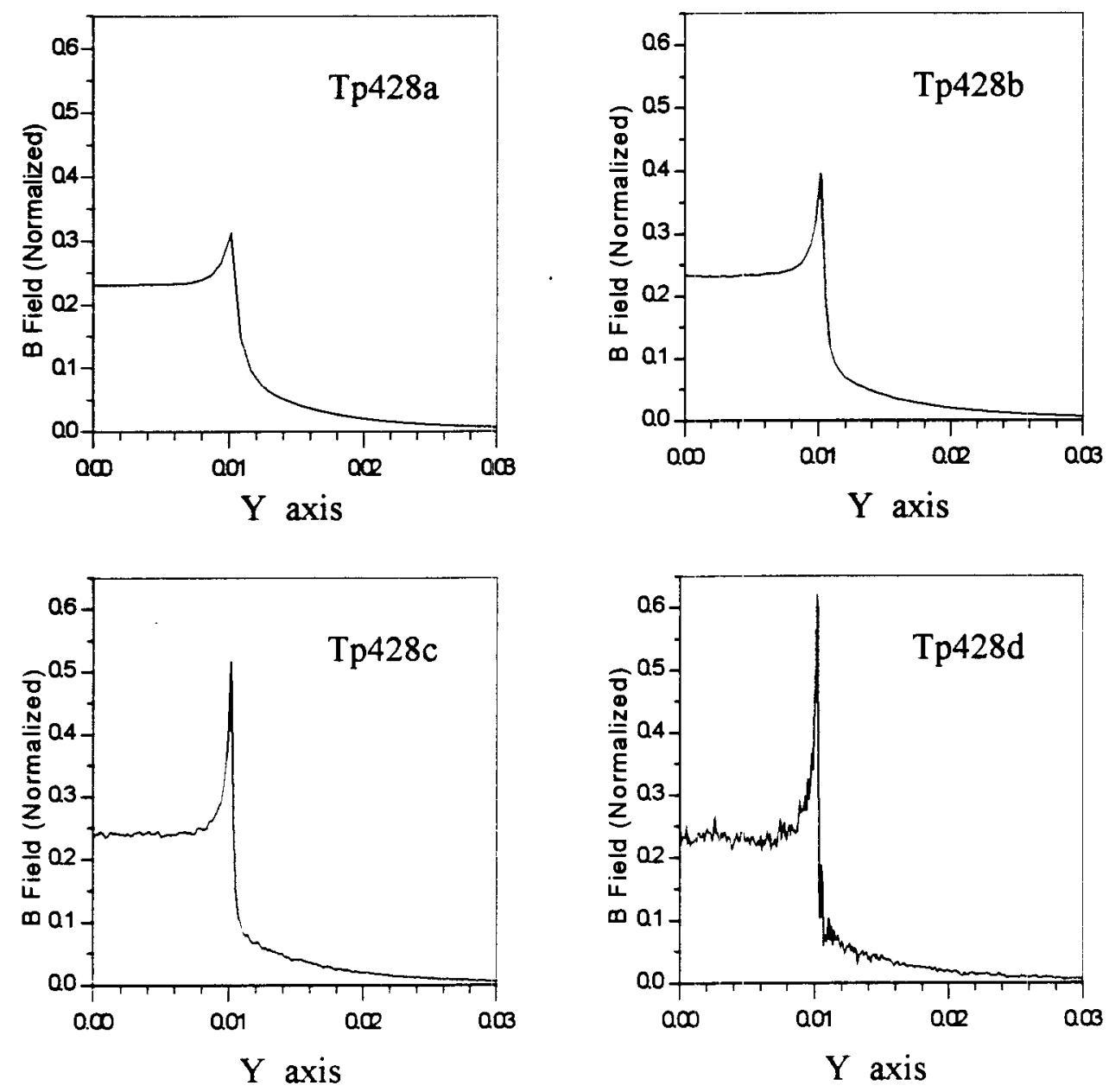

Fig. 2 The magnetic field along the nearest mesh line of the surface, figured out from MAFIA with program: (1) tp428a; (2) tp428b; (3) tp428c; (4) tp428d 
Fig. 1 shows a quasi-uniform mesh structure (roughly the same as that in row 6). It has only 4 lines inside the tape gap. The metal surface is on $4^{\text {th }}$ line, on which the magnitude of magnetic field is zero in MAFIA solution. The field for calculating the loss is that on the $3^{\text {rd }}$ line.

The field distribution along the 3rd line is shown in Fig.2 (1). Because of the coarse mesh, conceivably, it may cause unreliable results.

Fig. 2 demonstrates that the field has a peak near the tape edge. The denser mesh implies the points are closer to the metal surface, and the peak is sharper. Theoretically, right on the surface, the field will approach to infinitive on the edge. It is a singularity. This explains why MAFIA produces more error when the points are closer to the surface. It is then believed that MAFIA can not handle singularities.

\section{CONFORMAL MAPPING}

The surface current density is proportional to the magnitude of magnetic field on the surface; the loss is proportional to its absolute value square. Our problem is to figure out the magnetic field pattern around the edge of the tape.

It is well known 2-dimensional static field can be solved using conformal mapping, especially, if the electrodes form a polygon, Schwarz-Christoffel mapping ${ }^{{ }^{*}}$ can be applied. The magnetic field in our case is the same as a static field because it is a TEM mode. At first step, let's consider the case of infinite thin tape.

\section{Infinite thin tape mapping}

Fig.3 shows the conception of mapping. $z(x, y)$-plane involves two parallel electrodes that represent a uniform capacitance, so all force lines and equi-potencial lines are straight and uniform. $w(u, v)$-plane involves electrodes similar as in our case, that the top electrode represents a tape with edge and the bottom electrode ( $u$-axis) represents the symmetric axis, similar as the vertical axis in Fig. 1 . In the $w$-plane, the curves of " $x=$ const" represent force lines, " $y=$ const" represent equi-potential lines.

The mapping extends to infinitive. However, only the field in the vicinity of the edge is of concern. That part far from the edge, where the field is uniform, has little influence on the field near the edge. It is conceivable the field near the edge is a good representation for a tape of limited length.

This mapping is known to be represented by the following equation.

$$
w=z+e^{z}+1,
$$

Its components are:

$$
\begin{aligned}
& u=x+e^{x} \cos y+1 \\
& v=y+e^{x} \sin y
\end{aligned}
$$

" It can be found in many lecture books or from web side, e.g. http://www.ecs.fullerton.edu/ mathews/fofz/schwarz/schwarz.html 
The magnitude of field is the gradient of potential, therefore we have

$$
B x \nabla y=\frac{\partial y}{\partial u} i_{u}+\frac{\partial y}{\partial v} i_{v}
$$

At electrode $\partial y / \partial u=0$, therefore (ignored a constant factor)

$$
B=\left|\frac{\partial y}{\partial v}\right|
$$

To evaluate $B$, after algebraic manipulation one get an implicit function ${ }^{* 1}$

$$
\frac{\partial y}{\partial v}=\frac{\sqrt{f_{a}} \tan y+f_{b}}{\sqrt{f_{a}} \tan y+f_{b}+2 f_{b}(v-y) / \sin 2 y}
$$

where

$$
\begin{aligned}
& f_{a}=e^{2 x}-(u-x-1)^{2} \\
& f_{b}=e^{2 x}+u-x-1
\end{aligned}
$$

Most concerned is the integral along the surface of electrode of current density $j{ }^{* 2}$, whose magnitude is proportional to magnetic field. An easier approach can be applied.

According to the nature of analytic (holomorphic) function, its derivatives from different approaches will be equivalent, i.e.

$$
\frac{d z}{d w}=\frac{\partial z}{\partial u}=-i \frac{\partial z}{\partial v}=-i \frac{\partial x}{\partial v}+\frac{\partial y}{\partial v}
$$

From Fig. 3 one can see the force line $(x=$ const line $)$ is perpendicular to the electrode, so $\partial x / \partial v=0$. Therefore on the surface of electrode one has:

$$
\frac{d z}{d w}=\frac{\partial y}{\partial v}
$$

The current density square on the metal surface is (omit a constant factor)

$$
j^{2}=\left(\frac{\partial y}{\partial v}\right)^{2}=\left(\frac{d z}{d w}\right)^{2}
$$
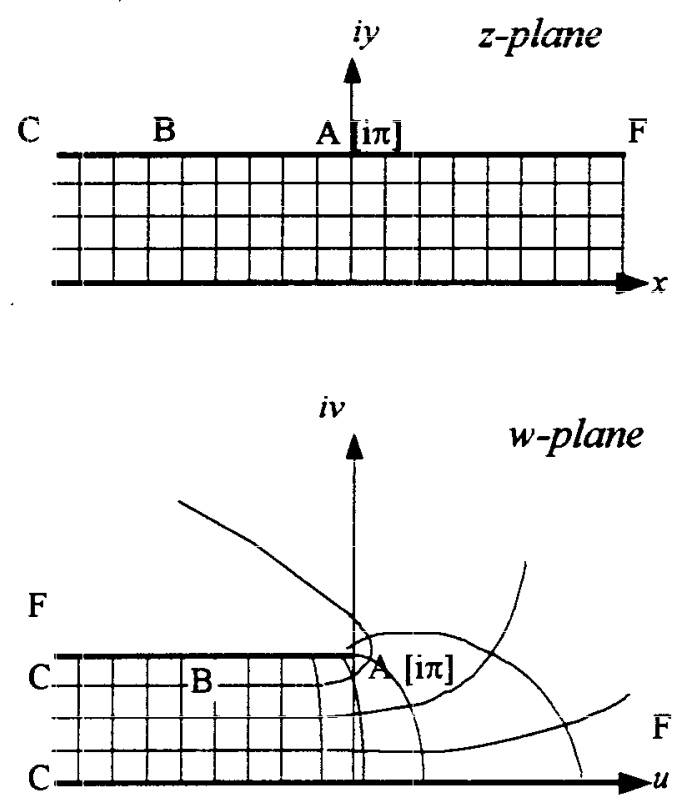

The power loss along $C A$ is (omit a constant factor):

$$
I_{\text {loss }}=\int_{C A} j^{2} d u=\int_{C A}\left(\frac{d z}{d w}\right)^{2} d w=\int_{C A} \frac{d z}{d w} d z
$$

\footnotetext{
$\because$ See Appendix A for detail.

" Instead of $i$. we symbol $j$ as current density here. in order to avoid the confusion with symbol of imaginary.
} 
From (1) we have: $\quad \frac{d w}{d z}=1+e^{z} \quad$ or $\quad \frac{d z}{d w}=\frac{1}{1+e^{z}}$

Along the path $C A, \quad z=x+i \pi, \quad$ so $\quad e^{z}=-\epsilon^{x}$ and $d z=d x$,

path $C A$ corresponds to $x=-x_{0}$ to $x=0$, then from (9) we obtain:

$$
I_{\text {loss }}=\int_{C A} j^{2} d u=\int_{-x_{0}}^{0} \frac{1}{1-e^{x}} d x=x-\left.\ln \left(1-e^{x}\right)\right|_{x_{0}} ^{p}
$$

The edge is at $\boldsymbol{x}=\boldsymbol{0}$. This integral is divergent.

This concludes that for a zero thickness tape, the current loss is infinitive. Thus we must apply a finite thickness to estimate the real loss.

\section{Finite thickness tape mapping}

The conformal mapping is done as follows. At first step, transform $w$-plane (shaded area, the top figure of Fig.4) to $\zeta$-plane (the shaded top half plan, middle figure); second step transform $\zeta$-plane to z-plane (shaded strip zone, bottom figure); and then combine both.

According to Schwarz Mapping,w-plane mapping onto $\zeta$-plane takes the following integration:

$$
w=K_{1} \int\left(\zeta-\xi_{A}\right)^{1 / 2}\left(\zeta-\xi_{B}\right)^{1 / 2} \zeta^{-1} d \zeta+K_{2}
$$

$\zeta$-plane mapping onto z-plane takes:

$$
\zeta=e^{z}
$$

After integrating, finally we get ${ }^{* 1}$ :

$$
w=z+f_{1}(z)+(1+t) \ln f_{2}(z)-\ln f_{3}(z)+K
$$

where

$$
\begin{aligned}
& f_{1}(z)=\sqrt{e^{2 z}+2 b e^{z}+1} \\
& f_{2}(z)=f_{1}(z)+e^{z}+t+1 \\
& f_{3}(z)=f_{1}(z)+b e^{z}+1 \\
& K=-\frac{1}{2}(b-1) \ln \left(b^{2}-1\right) \\
& b=I+t
\end{aligned}
$$

where $t$ is the relative thickness of the tape, which can be seen from Fig.4. The coordinates of the points $\mathrm{A}$ and $\mathrm{B}$ in $\zeta$ - and $z$-plane are as follows:

$$
\xi_{A}=-b-\sqrt{b^{2}-1}
$$
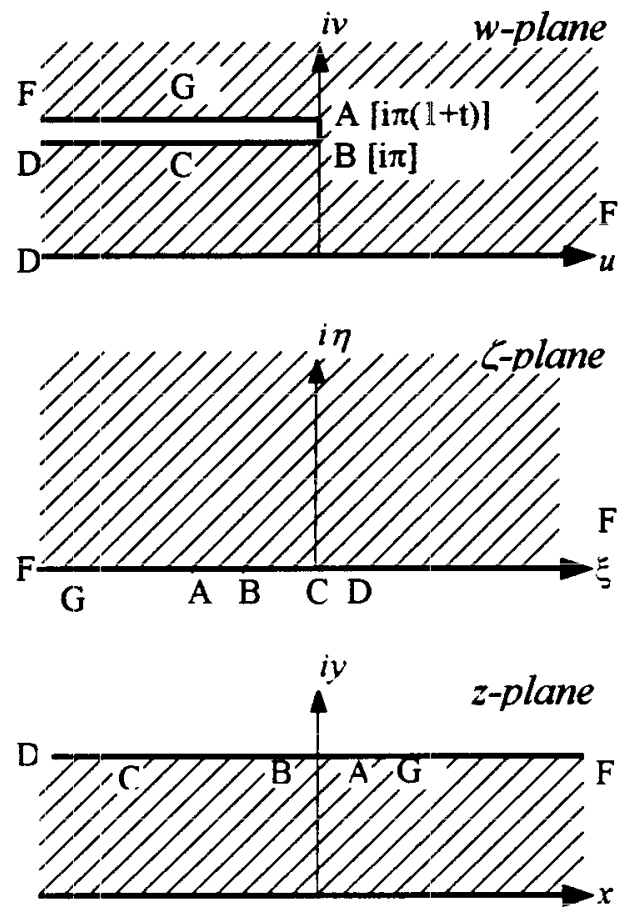

Fig. 4 Schwarz Mapping of finite tape

\footnotetext{
${ }^{-1}$ See Appendix $\mathrm{B}$ for detail
} 


$$
\begin{aligned}
\xi_{B} & =-\dot{b}+\sqrt{b^{2}-i} \\
z_{A} & =x_{A}+i y_{A}=\ln \xi_{A} \\
& z_{B}=x_{B}+i y_{B}=\ln \xi_{B} \\
\text { or } \quad x_{A} & =\ln \left|\xi_{A}\right|, \quad y_{A}=\pi \\
& x_{B}=\ln \left|\xi_{B}\right|, \quad y_{B}=\pi
\end{aligned}
$$

Above formula (12) and (13) determine the field pattern.

Similar process can be applied to evaluate the power loss. It is known that:

$$
\boldsymbol{B} \propto \frac{d z}{d w}
$$

This is applicable for all segments $C-B, B-A, A-G$. From (10), (11), we obtain:

$$
\frac{d w}{d z}=\frac{d w}{d \zeta} \cdot \frac{d \zeta}{d z}=\frac{\sqrt{\left(\zeta-\xi_{A}\right)\left(\zeta-\xi_{B}\right)}}{\zeta} \cdot \zeta=\sqrt{\left(\zeta-\xi_{A}\right)\left(\zeta-\xi_{B}\right)}=\sqrt{\zeta^{2}+2 b \zeta+1}
$$

Substituting (11), then $\quad \frac{d z}{d w}=\frac{1}{\sqrt{e^{2 z}+2 b e^{z}+1}}$

At $z$-plane, the loss integral is along $C-B-A-G$, where $z=x+i \pi$, so

$$
e^{z}=e^{x+i \pi}=-e^{x}
$$

Thus from (15) $\quad\left(\frac{d z}{d w}\right)^{*}=\left(\frac{d z}{d w}\right)$

or $\quad\left|\frac{d z}{d w}\right|^{2}=\left(\frac{d z}{d w}\right) \cdot\left(\frac{d z}{d w}\right)^{*}=\left(\frac{d z}{d w}\right)^{2}$

Then we have the loss integral ${ }^{* 1}$ :

$$
\left|\int B \cdot B^{*} d w\right| \propto\left|\int\left(\frac{d z}{d w}\right)^{2} d w\right|=\left|\int\left(\frac{d z}{d w}\right) \cdot d z\right|
$$

Note that (18b) is valid only on the surface (i.e. the lines $C B, B A, A G$ ), not everywhere. It is also worthy of note that though $\left(\frac{d z}{d w}\right)$ and $\left(\frac{d z}{d w}\right)^{2}$ are analytic (holomorphic) functions, but $\left|\frac{d z}{d w}\right|^{2}$ is not, because absolute value function is a real function, it can not satisfy the CauchyRiemann equation. Therefore, the integral (19) is not path independent, i.e. the path must be along the surface $C B, B A, A G$. Besides, it can be seen from formula (13) and (15) that points $A$

\footnotetext{
"The absolute value symbol is added because at segment $B A, d w=i d v$ is imaginary, and so is $d z / d w$.
} 
and $B$ are singularities that the denominator in (16) is zero. The integral must be taken piecewise, i.e. takes three separate integrals. Nevertheless, they are integrable. From (16), (17) and (19) the loss integral has the form:

$$
I_{W}=\left|\int \frac{d x}{\sqrt{e^{2 x}-2 b e^{x}+1}}\right|
$$

Assume $y=e^{x}, d x=d y / y$ then:

$$
I_{w}=\left|\int \frac{d y}{y \sqrt{y^{2}-2 b y+1}}\right|
$$

This is ready to be carried out by integral table.

\section{Numerical solution}

Let's consider the real case. The thickness of the tape is $0.15 \mathrm{~mm}$. A haif of tape width, i.e. $W$ in Fig. 1 , is $10.16 \mathrm{~mm}$. A half of the gap width, i.e. $g$ in Fig. 1 , is $3.12 \mathrm{~mm}$. From Fig.4, $t$ is the relative thickness in comparison with $g$, thus:

$$
\begin{aligned}
& t=\frac{0.15}{3.12}=0.04808 \\
& b=I+t=1.04808
\end{aligned}
$$

The lower limit of the integral in the w-plane, or point $C$, should be an appropriate depth in comparison with the gap width, or $\pi$ in Fig.4. Thus we have:

$$
u_{C}=-10.16 \times \frac{\pi}{3.12}=-10.23
$$

where $u_{C}$ is the coordinate $u$ of point $C$ on $w$-plane. From Fig. $4, u_{G}=u_{C}=-10.23$. It means when the loss is being calculated, the surface on the left of $u_{G}$ and $u_{C}$ will be ignored.

The integral at $w$-plane should be taken along the metal surface, $C-B-A-G$ in the top figure of Fig.4. The counterpart on $z$-plane (bottom figure) will be along $C-B-A-G$ too. From (11) to (13) we obtain their coordinates listed in following table.

Table 2

\begin{tabular}{|c|l|l|l|}
\hline Point & z-plane & S-plane & w-plane \\
\hline $\mathrm{A}$ & $x_{A}=0.309$ & $\xi_{A}=-1.362$ & $u_{A}=0, v_{A}=\pi(1+t)=3.293$ \\
\hline $\mathrm{B}$ & $x_{B}=-0.309$ & $\xi_{B}=-0.734$ & $u_{B}=0, \quad v_{B}=\pi$ \\
\hline $\mathrm{C}$ & $x_{C}=-11.344$ & $\xi_{C}=-1.18 \mathrm{E}-5$ & $u_{C}=-10.23, v_{C}=\pi$ \\
\hline $\mathrm{G}$ & $x_{G}=+2.7$ & $\xi_{G}=-14.88$ & $u_{G}=-10.23, v_{G}=3.293$ \\
\hline
\end{tabular}

The integral (19) can be figured out by virtue of integral table, with appropriate attention to the sign of the primitives. They are: 
On segment $C-B$,

$$
I_{W-C B}=x-\left.\ln \left(1-b e^{x}+\sqrt{e^{2 x}-2 b e^{x}+1}\right)\right|_{x_{C}} ^{x_{B}}=13.192
$$

On segment $B-A$,

$$
I_{W-B A}=\arcsin \left(\frac{b e^{x}-1}{e^{x} \sqrt{b^{2}-1}}\right)_{x_{B}}^{x_{A}}=3.1416
$$

On segment $A-G$,

$$
I_{T-A G}=x-\left.\ln \left(b e^{x}-1-\sqrt{e^{2 x}-2 b e^{x}+1}\right)\right|_{x_{1}} ^{x_{G}}=1.803
$$

Finally we get total relative loss, the sum of above, is $I_{W}=18.137$.

Note this is a relative value, in order to be comparable, we choose a reference such that the field is completely uniform. From (16), at uniform region,

when $\quad x<0$ and $|x|>>1, \quad \sqrt{e^{2 x}-2 b e^{x}+1} \approx 1$

So, $\quad I_{\text {ref }}=\int_{C B} 1 d w=u_{B}-u_{C}=10.23$

As a comparison,

$$
\frac{\int B^{2} d u}{\int B_{0}^{2} d u}=\frac{18.137}{10.23}=1.773
$$

It means that due to the non-uniformity of the edge current, the loss is $77 \%$ enhanced in comparison with a uniform case.

\section{COMPARISON WITH MAFLA RESULT}

In order to make a sensible comparison, we should use same reference. That is, we should figure out how much enhancement of a non-uniform case to a uniform case in MAFIA simulation.

Fig. 2 and Table 1 show that the loss is strongly dependent on the mesh chosen. At section "Introduction", the data are quoted from a 3-dimentional simulation, of which the field around its center part is close to that of Program tp423, showed in row \#1 on Table 1 , also very close the pattern of tp428a shown in Fig.2 (1). We quoted the field data of tp423 and integrated numerically. It indicated an enhancement as follows:

$$
\frac{\int B^{2} d u}{\int B_{0}^{2} d u}=\frac{11.02}{10.16}=1.085
$$

In comparison with the result of conformal mapping, the difference is: 


$$
\frac{1.773}{1.085}=1.63
$$

This implies that the MAFIA using a moderate mesh may cause an underestimation on loss about $63 \%$. Or the $Q$ value may be $63 \%$ higher than the real. This is very close to the measured date.

\section{CONCLUSION}

Many efforts were made for improving the accuracy of the beryllium conductivity test. However, the absolute value test based on MAFIA simulation indicated too much error. From conformal mapping analysis it concludes that in the case of existing sharp edge, MAFIA dealing with loss calculating will cause significant error, which can be as high as $60 \%$ or more. The analysis interprets reasonably the discrepancy between measured data and MAFIA prediction. For a real measurement, relative data would be more reliable rather than an absolute data based on the simulation prediction if a strict theoretic prediction were not available.

\section{References}

[1] Yongxiang Zhao, Haipeng Wang, "Experiment of electrical conductivity at low temperature (preliminary measurement)", BNL-65629, CAP-221-MUON-98R, July 1998.

[2] Yongxiang Zhao, Haipeng Wang, "The measurement of the properties of beryllium foil". BNL, CAP-263-MUON-99.

\section{APPENDIX A (Conformal Mapping of infinite thickness tape)}

According to Schwarz-Christoffel mapping, the mapping of Fig. 3 is known to be:

$$
w=z+e^{z}+1
$$

from which we have: $\left\{\begin{array}{c}u=x+e^{x} \cos y+1 \\ v=y+e^{x} \sin y\end{array}\right.$

$$
\text { From (A2), } \quad x=u-1-\frac{v-y}{\tan y}
$$

Since $0<y<\pi \sin y>0$, from (A2), always $v-y>0$. From (A2), we also get:

$$
\begin{aligned}
& v-y=\sqrt{e^{2 x}-(u-x-1)^{2}} \\
& \text { or } \quad y=v-\sqrt{f_{a}} \\
& \text { where } \quad f_{a}=e^{2 x}-(u-x-1)^{2} \\
& \text { From (A5a) } \frac{\partial y}{\partial v}=1-\frac{1}{2 \sqrt{f_{a}}} \frac{\partial f_{a}}{\partial v}
\end{aligned}
$$


From (A5b) $\quad \frac{\partial f_{a}}{\partial v}=2 f_{b} \frac{\partial x}{\partial v}$

where

$$
f_{b}=e^{2 x}+u-x-1
$$

From (A3) $\quad \frac{\partial x}{\partial v}=\frac{\partial y}{\partial v}\left(\frac{1}{\tan y}+\frac{v-y}{\sin ^{2} y}\right)-\frac{1}{\tan y}$

Substituting (A8) to(A7) and (A6), we get:

$$
\frac{\partial y}{\partial v}=1-\frac{f_{b}}{\sqrt{f_{a}}} \frac{1}{\tan y}-\frac{f_{b}}{\sqrt{f_{a}}} \frac{\partial y}{\partial v}\left(\frac{1}{\tan y}+\frac{v-y}{\sin ^{2} y}\right)
$$

Shift the item which includes $\partial y / \partial v$ to the left, then we finally obtain the equation (5a)

$$
\frac{\partial y}{\partial v}=\frac{\sqrt{f_{a}} \tan y+f_{b}}{\sqrt{f_{a}} \tan y+f_{b}+2 f_{b}(v-y) / \sin 2 y}
$$

\section{APPENDIX B (Conformal Mapping of finite thickness tape)}

According to Schwarz-Christoffel mapping, the top figure of Fig.4 can be regarded as a polygon, of which the three angles are $\alpha_{A}=\frac{3}{2} \pi, \alpha_{B}=\frac{3}{2} \pi, \alpha_{D}=0$ respectively. Then the mapping from $w$-plane to $\zeta$-plane takes the form:

$$
\begin{aligned}
& \left.w(u, v)=K_{1} \int\left(\zeta-\xi_{A}\right)^{\left(\frac{\alpha_{A}}{\pi}-1\right)}\left(\zeta-\xi_{B}\right)^{\left(\frac{\alpha_{B}}{\pi}-1\right)}\left(\zeta-\xi_{D}\right)\right)^{\left(\frac{\alpha_{D}}{\pi}-1\right)} d \zeta+K_{2} \\
& \text { or } \quad w(u, v)=K_{1} \int\left(\zeta-\xi_{A}\right)^{1 / 2}\left(\zeta-\xi_{B}\right)^{1 / 2} \zeta^{-1} d \zeta+K_{2}
\end{aligned}
$$

where $\xi_{A}$ and $\xi_{B}$ are the coordinate of points $A$ and $B$ at the real axis on $\zeta$-plane. $\xi_{D}=0$ is chosen arbitrarily. Above integral can be divided into three integrals, of which the solution can be found in handbook:

$$
\text { where } \quad \begin{aligned}
w & =K_{1} \int \frac{\sqrt{\left(\zeta-\xi_{A}\right)\left(\zeta-\xi_{B}\right)}}{\zeta} d \zeta+K_{2}=K_{1} \int \frac{\zeta^{2}+\beta \zeta+\gamma}{\zeta \sqrt{\zeta^{2}+\beta \zeta+\gamma}} d \zeta+K_{2} \\
& =K_{1}\left(I_{1}+I_{2}+I_{3}\right)+K_{2} \\
\zeta^{2} & +\beta \zeta+\gamma=\left(\zeta-\xi_{A}\right)\left(\zeta-\xi_{B}\right) \\
\beta & =-\left(\xi_{A}+\xi_{B}\right) \\
\gamma & =\xi_{A} \xi_{B}>0
\end{aligned}
$$

The three integrals are:

$$
I_{1}=\int \frac{\zeta}{\sqrt{\zeta^{2}+\beta \zeta+\gamma}} d \zeta=\sqrt{\zeta^{2}+\beta \zeta+\gamma}-\frac{1}{2} I_{2}
$$




$$
\begin{aligned}
& I_{2}=\int \frac{\beta}{\sqrt{\zeta^{2}+\beta \zeta+\gamma}} d \zeta=\beta \ln \left(2 \zeta+\beta+2 \sqrt{\zeta^{2}+\beta \zeta+\gamma}\right) \\
& I_{3}=\int \frac{\gamma}{\zeta \sqrt{\zeta^{2}+\beta \zeta+\gamma}}=-\sqrt{\gamma} \ln \left(\frac{\sqrt{\zeta^{2}+\beta \zeta+\gamma}+\sqrt{\gamma}}{\zeta}+\frac{\beta}{2 \sqrt{\gamma}}\right)
\end{aligned}
$$

Thus: $\quad w=K_{1}\left[f_{1}+\frac{\beta}{2} \ln \left(2 \zeta+\beta+2 f_{1}\right)-\sqrt{\gamma} \ln \left(\frac{f_{1}+\sqrt{\gamma}}{\zeta}+-\frac{\beta}{2 \sqrt{\gamma}}\right)\right]+K_{2}$

where

$$
\begin{aligned}
& f_{1}=\sqrt{\left(\zeta-\xi_{A}\right)\left(\zeta-\xi_{B}\right)}, \quad \xi_{A}<\xi_{B}<0 \\
& f_{1}=\left\{\begin{array}{lc}
0 & \zeta=\xi_{A}, \text { or } \zeta=\xi_{B} \\
\text { real } & \zeta<\xi_{A}, \text { or } \zeta>\xi_{B} \\
\text { image } & \xi_{A}<\zeta<\xi_{B}
\end{array}\right.
\end{aligned}
$$

Assume: $b=-\frac{1}{2}\left(\xi_{A}+\xi_{B}\right)=\beta / 2 \quad>0$

$$
\begin{array}{ll}
c=\sqrt{\gamma}=\sqrt{\xi_{A} \xi_{B}} & >0 \\
d=\xi_{B}-\xi_{A} & >0
\end{array}
$$

Then: $\quad w=K_{1}\left[f_{1}+b \ln 2\left(\zeta+b+f_{1}\right)-c \ln \left(\frac{f_{1}+c}{\zeta}+\frac{b}{c}\right)\right]+K_{2}$

In order to determine $\xi_{A}, \xi_{B}$ and the constants $K_{1}$ and $K_{2}$, we substituting the following conditions:

$\begin{array}{cll}\text { Point A: } & w_{A}=i \pi(1+t), & \zeta=\xi_{A} \\ \text { Point B } \quad w_{B}=i \pi & \zeta=\xi_{B} \\ \text { One constant is arbitrary, we choose: } & K_{1}=1\end{array}$

and then found: $\quad b=1+t$

$c=1$

$$
\begin{aligned}
& K_{2}=-\frac{1}{2} t \ln \left(2 t+t^{2}\right)-(1+t) \ln 2 \\
& \xi_{A, B}=-b \mp \sqrt{b^{2}-1}
\end{aligned}
$$

Finally we get:

$$
w=\ln \zeta+f_{1}(\zeta)+(1+t) \ln f_{2}(\zeta)-\ln f_{3}(\zeta)-\frac{t}{2} \ln \left(2 t+t^{2}\right)
$$

where

$$
\begin{aligned}
& f_{1}=\sqrt{\zeta^{2}+2(1+t) \zeta+1} \\
& f_{2}=f_{1}(\zeta)+\zeta+1+t \\
& f_{3}=f_{1}(\zeta)+\zeta+1+t \zeta
\end{aligned}
$$

The mapping from $\zeta$-plane to $z$-plane can be found from lecture books that

$$
z=\ln \zeta, \text { or } \zeta=e^{z}
$$

Substituting (B10) into (B9) we get equation (12), and consequently (13). 